\title{
Hierarchical stages in the processing of visual information
}

\author{
JAMES E. HOFFMAN \\ University of Delaware, Newark, Delaware 19711
}

\begin{abstract}
Previous research had indicated that when subjects are instructed to report one of a number of visually displayed items, both the number and spacing of the presented material affect report accuracy and latency. The present experiment sought to determine the nature and temporal course of the interference provided by nonattended visual material. Subjects reaction times were measured for deciding which of two targets occupied the indicated position in one or eight element displays. Placing replicas of the target in nonindicated display positions was equivalent to presenting the target alone. Members of the opposite response set produced maximum interference, while encodable and unencodable noise elements not belonging to a response set produced an intermediate decrement. For all display types, presenting the indicator prior to display onset decreased reaction time. Dividing each of the display elements into two parts and presenting the parts asynchronously provided evidence that subjects were indifferent to the presence of complete forms for the first $50 \mathrm{msec}$. These results were interpreted as supporting the existence of a hiararchical sequence of stages consisting of a preattentive stage which segregates the input into objects and an attentive stage which is likened to a spatial scanner responsible for synthesizing the crude preattentive features into recognized forms. The concurrent operation of these stages provides for the redirection of attention when changes in the input are detected.
\end{abstract}

One aspect of intelligence in higher organisms is the ability to assign an invariant response to a stimulus undergoing changes in shape. Attempts to endow computers with similar pattern recognition capabilities have rsulted in hierarchical systems which first attempt to segregate the input into objects on the basis of "local" cues (Guzman, 1969) and secondly to recognize each object by matching its features with a stored list.

Neisser (1967) has summarized the evidence for a similar structure underlying visual information processing in the human observer. A global, parallel, preattentive stage segregates and groups the visual input on the bases of proximity, similarity, and other Gestalt factors. Attentional processes, which are limited in capacity, are responsible for providing a detailed description of the first stage output.

Despite Neisser's (1967) compelling arguments, there is little direct evidence for such a hierarchical scheme. Eriksen and Spencer (1969) and Shiffrin and Gardner (1972) showed that subjects can search an array of characters for a target equally well whether the characters are presented simultaneously or sequentially. The important variable appears to be the similarity between target and noise. This finding has led to a number of models which suppose that display features are extracted in a parallel, unlimited-

This work is based on a doctoral dissertation submitted to the University of Illinois in partial fultillment of the requirements for a PhD degree. This research was supported by United States Public Health Service Research Grant MH-1206 and Career Program Award K6-MH-22014 to Charles W. Eriksen. capacity fashion with false alarms provided by confusable noise elements (Gardner, 1973; Shiffrin \& Geisler, 1973). Selective allocation of processing resources is then presumed to occur at a late stage in the processing sequence, perhaps in the choice of what material to rehearse in short-term memory.

Another ine of research, however, suggests that the human observer may select which aspect of a stimulus array will undergo detailed processing. Sperling (1960) showed that a cue designating one row of a multielement display was effective in improving the report accuracy of that row. The stage at which this selection occurs must be prior to short-term memory, since Spencer (1969) found that the range of delays over which a mask was effective could be lengthened by delaying the partial report cue. That is, the cue appeared to control the speed at which a given element in iconic memory was identified.

Research with the partial cue paradigm has shown that attention devoted to a particular display element does not preclude processing of other display elements. Those characters within about $1 \mathrm{deg}$ of visual angle of the target have an opportunity to be processed to the point of identification and produce interference if they evoke a response incompatible with that of the target (Eriksen \& Hoffman, 1972, 1973; Holmgren, 1974).

Although characters in adjacent positions to the target may produce response competition, this is not the major source of interference. Even if the display is composed of characters belonging to the same response set, target identification time will be slower than if the target had been presented alone (Eriksen \& Hoffman. 1973). This effect can be largely eliminated 


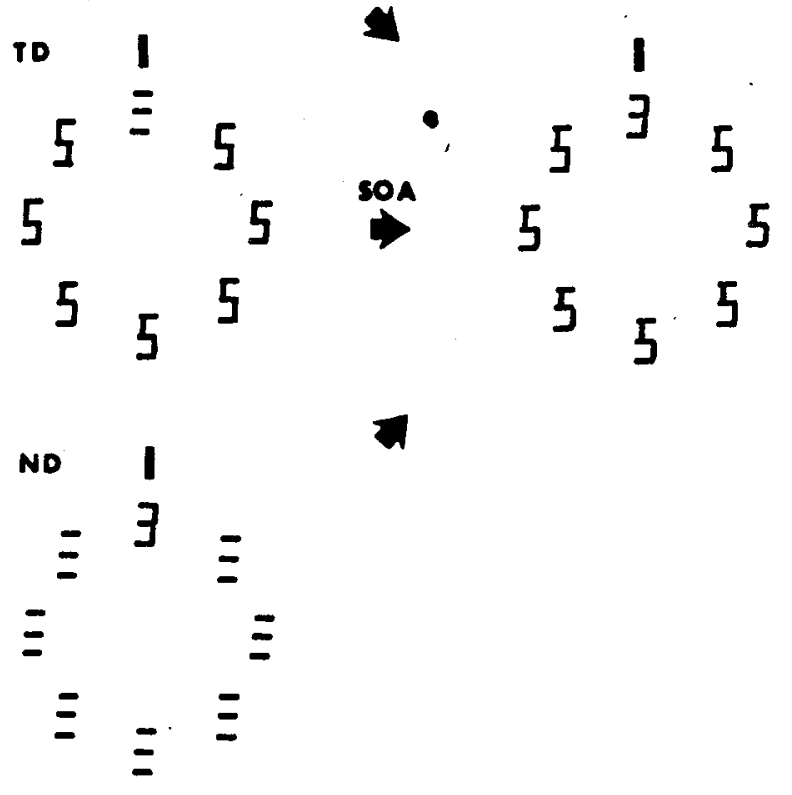

Figure 1. A schematic representation of the three sequence types employed: leading indicator (LI), target delayed (TD), and noise delayed (ND).

by presenting the spatial cue approximately $200 \mathrm{msec}$ prior to onset of the display information.

These results fit within a scheme that supposes that a display is processed at several levels. Early in the processing sequence, a global description of the display is attempted. This description may take the form of designating how many objects are present, whether they form groups, etc. Certainly, subjects can convey this information even though not instructed to do so (Eriksen \& Rohrbaugh, 1970).

Next, the level of description is shifted to the object level so that a particular spatial region of the input is processed faster than nonattended regions. The allocation of processing capacity takes time which can be preprogrammed by presenting the cue prior to display onset.

The present experiment was initiated to provide more direct information on the hierarchy of processing stages. The method is illustrated in Figure 1. The leading indicator condition (LI) provides data on the efficiency of attention for a number of different types of noive elements, as shown in Figure 2. The response-compatible (RC), responseincompatible (RI), and single-target (S) conditions provide replication of conditions included in the Eriksen and Hoffman (1973) report. The neutral (N) and character-like $(\mathrm{C})$ conditions provide information on the role of verbal labels in selective attention. If encodability per se is a determiner of interference, a difference between the $\mathrm{N}$ and $\mathrm{C}$ conditions should be observed. If response competition is the only source of interference, they should be equivalent to the RC and $S$ conditions.

In the noise-delayed condition (ND), segments completing the noise elements are shown at various times after onset of the complete target and indicator. If the initial stages of perception do not involve extraction of symbolic information, but rather consist of a segmenting of the input and programming of an attentional mechanism, the time required to process the target or indicated element should be invariant, with short delays of the segments defining particular noise elements. At some point, the processing will shift to the level of individual display elements and a delay of noise element segments will become important.

Delaying target element seyments should provide converging evidence on the duration of a preattentive stage. In addition, it should furnish information on the mechanism by which attention operates. For example, one could imagine attention as a simple increase in processing speed so that attended elements finish first and enjoy the benefits of immediate transfer to short-term memory, Delaying the target in this case would be detrimental since noise elements would receive a "head start."

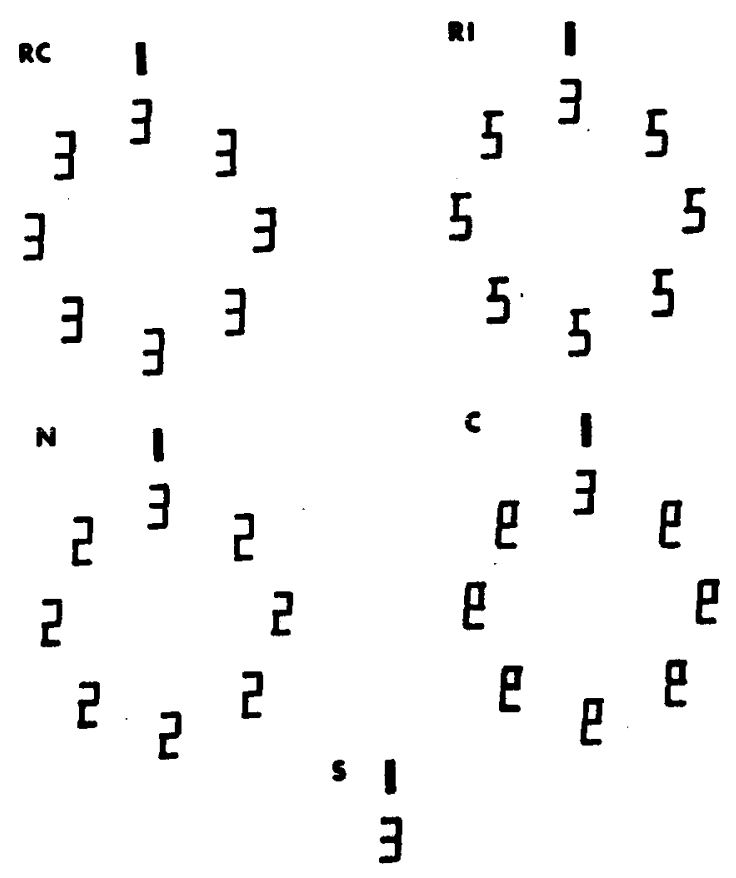

Figure 2. The target element embedded in the types of noles: responve compatible ( $R C)$, response lncompatible (RD, neatral $(N)$, character-Hke (C), and aingle target (S). 


\section{METHOD}

\section{Subjects}

Four University of Illinois students (one male) served as paid subjects. A tifth subject was dismissed because of high error rate in the practice sessions.

\section{Apparatus and Stimuli}

Stimuli were displayed in a Scientific Prototype Model GA three-field tachistoscope modified by replacing the manufacturer's bulbs with Sylvania F4T5/CWX fluorescent lamps. All three fields were set and maintained at $4 \mathrm{tl}$, as measured with a Spectra spot photometer. A black tixation cross, subtending $.17 \times .17 \mathrm{deg}$ of visual angle, appeared in the center of the adaptation field, which remained on at all times except when Field II was activated.

Subjects initiated a triwi by closing a hand-held microswitch. The on pulse obtained from the switching logic of the tachistoscope was used to close a relay and start a Hunter Model 1522 digital clock set to time in milliseconds. No warning signal was employed.

A spring-loaded toggle switch, located under the subject's right hand, triggered one of two Hunter timers, stopping the clock and indicating the direction of response. Reaction time was recorded in milliseconds.

The stimuli consisted of an eight-element circular display centered on the fixation cross. ensuring that each element fell on an equally sensitive foveal area. The display subtended 2 deg of visual angle in diam with center-to-center intercharacter distance equal to .7 deg.

Each character was fornicd by combining various subsets of a seven-segment display. Individual segments consisted of the capital letter I obtained from Paratype No. 11316, subtending .17 deg in length. The resulting character was $.40 \mathrm{deg}$ in height and $.17 \mathrm{deg}$ in width.

Four cards were constructed consisting of the three horizontal segments in each of the eight positions with a bar indicator at the $12,3,6$, or $90^{\circ}$ clock position. The bar was $.28 \times .08 \mathrm{deg}$ positions with its near end. $.40 \mathrm{deg}$ from the center of the indicated character. The indicator was placed so as to lie on an imaginary radius through the character. Four additional cards were constructed containing horizontal bars only in the indicated position.

A second set of cards was constructed by placing vertical segments on a clear plastic card. The combination of display cards resulted in a display containing either one or eight characters. In all cases. the character indicated by the bar was a 3 or a 5 . The noise elements were of five types: response compatible $(\mathrm{RC})$, in which noise elements were the same as the indicated letter; response incompatible ( $\mathrm{RI})$, in which elements belonged to the response class opposite that of the target; neutral (N), in which the element (2) belonged to neither response class; a character-like element (C), which was a mirror reversal of a 9; and tinally, the case when the target appeared alone as a single element (S). Each of these display types is illustrated in Figure 2.

\section{Procedure}

The subject received display information in the three different orders illustrated in Figure 1. In the leading indicator condition (LI). the complete display was preceded by the bar indicator at 0 , $50,100,150$, or $200 \mathrm{msec}$ SOA. In the noise delayed condition (ND), the subject received target, indicator, and incomplete noise elements simultaneously followed at various SOAs by the necessary segments to complete the noise elements. In the target-delayed condition (TD), complete noise elements, ind icator, and incomplete target wers presented, followed by the necessary segments to complete the target as a 3 or a 5 . The delays used in the ND and TD conditions were the same as those employed in the LI sequence.

Each subject served in two practice sessions followed by 12 experimental sessions. Each experimental session was devoted to the $\mathrm{LI}, \mathrm{ND}$, or $\mathrm{TD}$ condition. The order of conditions across sessions was determined by forming a l.atin square for the first nine sessions and then repeating one of the orders for the last three sessions

Each session consisted of five blocks of $\mathbf{3 0}$ trials each, one block for each of the SOA values. Within a block, 3 and 5 occurred as targets in the context of each of the five types of noise three times each in a random order. Over sessions, each target occurred equally often with each of the tive noise types in each of the four target pusitions equally often. The order of SOAs within sessions was partially counterbalanced a ross sessions for each of the three sequence types.

Each session began with eight practice tulals at the first condition to be run that day. The subject was instructed to initiate a trial with his left hand when the fixation cross appeared in good focus and place his right hand on the response switch. When he had determined the identity of the indicated element, he was to move the lever right or left to indicate his choice of 3 or 5 . The assignment of targets to direction of response was counterbalanced across subjects. The subjects were instructed to respond as quickly as possible commensurate with high accuracy and were given RT feedback after each trial. For all conditions, the clock began counting as soon as a completed target was present. At initiation, the tixation field remained on with Field $I$ and terminated when Field II came on Fields I and II remained on together so that the total duration of Field I was $500 \mathrm{msec}$.

Error rate averaged over conditions and subjects was $3 \%$, varying from $8 \%$ for the RI condition to $1 \%$ for the RC condition. The remaining conditions showed a uniform error rate of $2 \%$. Error trials were rerun at random points within the same or later blocks, yielding $24 \mathrm{R}$ T's for each subject for each condition. Due to the small number of errors. RTs for these trials will not be discussed in the remainder of this paper.

\section{RESULTS}

Mean reaction times, averaged across subjects are shown in Figures 3-5 for the LI, ND, and TD conditions, respectively. A repeated measures analysis of variance on these data revealed significant main effecis of sequence type $[F(2,6)=6.56, p<.05]$, SOA $[F(4,12)=21.97, p<.001]$, and noise type $[F(4.12)=52.22, p<.001]$. All interactions were signiticant beyond the .01 level, except for Sequence Type by SOA, which was significant at $.025[\mathrm{~F}(8,24)$ $=3.16, \mathrm{p}<.025]$.

The data from the LI condition, summarized in Figure 3, show a consistent decrease in RT as the indicator leads the display. Also apparent is the effect of noise type. $\mathrm{RC}$ and $\mathrm{S}$ latencies are equivalent at all SOAs and are shorter than the $\mathbf{N}$ and $\mathbf{C}$ latencies which also appear to be equivalent. As anticipated on the basis of previous research (Eriksen \& Eriksen, 1974b; Eriksen \& Hoffman, 1973), the RI condition produced the maximum interference.

Analysis of variance on the data from the LI condition verified these inferences. Significant main effects were found for $\operatorname{SOA}[F(4,12)=10.32$, $\mathrm{p}<.001]$, noise type $[\mathrm{F}(4,12)=63.23, \mathrm{p}<.001]$, and SOA by Noise Type $[F(16,48)=2.68, \mathrm{p}<.004]$. A separate analysis conducted on the $R C$ and $S$ data showed a significant effect of $\operatorname{SOA}[F(4,12)=6.22$, $\mathrm{p}<.006]$, while noise type $[\mathrm{F}(1,3)<1]$ and SOA by Noise Type $[F(4,12)<1]$ were insignificant.

Data from the noise-delayed condition (ND) shown in Figure 4 reveal a relatively tlat $\mathrm{RT}$ function for a 


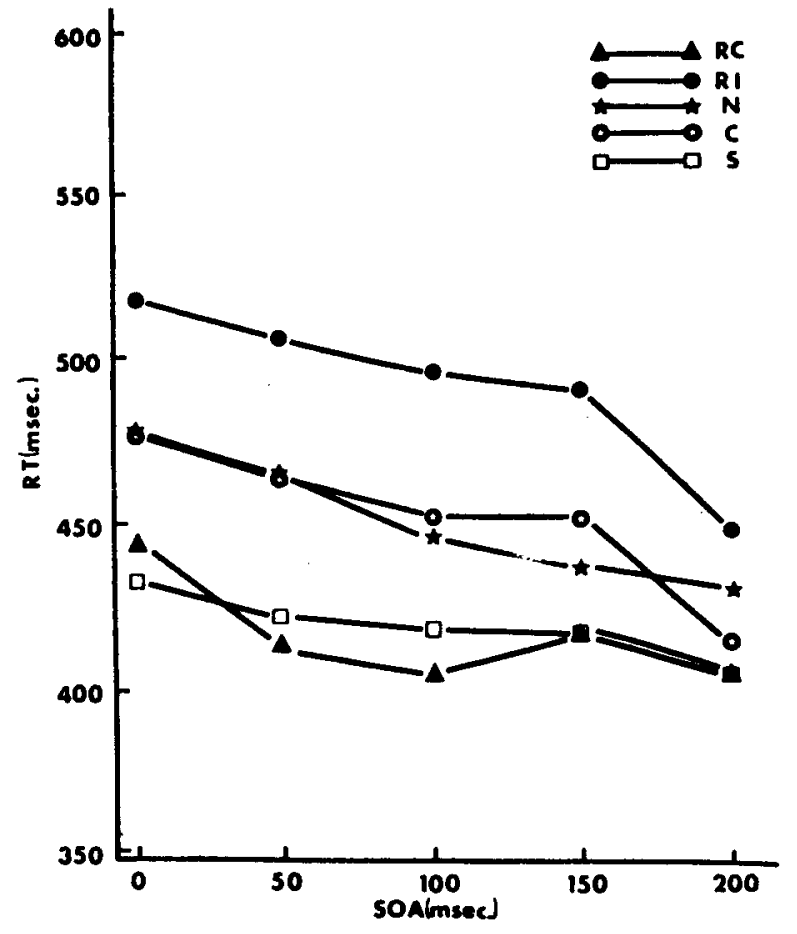

Figure 3. Mean reaction time as a function of SOA of the leading indicator and the display. Parameter is noise type.

50-nsec delay of noise segments, followed by a gradual decrease to the level of a single target by $200-\mathrm{msec}$ SOA. The effects of noise type are virtually identical to those shown in Figure 3. The $S$ condition, in which there are no noise elements to be delayed, represents a replication within each of the blocks devoted to a particular delay of the noise segments. It should have zero slope and, as the data show, this is the case.

An analysis of variance revealed significant effects of SOA $[F(4,12)=13.73, p<.001]$, noise $[F(4,12)=$ $30.48, \mathrm{p}<.001]$, and $\mathrm{SOA}$ by Noise $[\mathrm{F}(4,16)=8.26$, $\mathrm{p}<.001]$. A second analysis was conducted on the data from the 0 - and $50-\mathrm{msec}$ SOAs, which revealed a significant effect of noise $[F(4,12)=50.57$, $\mathrm{p}<.001]$; SOA $[\mathrm{F}(1,3)<1]$ and SOA by Noise $[F(4,12)=1.34, p<.311]$ were not significant. The small, but consistent, difference between the $R C$ and $S$ conditions apparent in Figure $\mathbf{4}$ was not significant $[F(1,3)=7.87, p<.06]$, nor were the effects of SOA $[F(4,12)<1]$ and Noise by SOA $[F(4,12)<1]$.

The data from the target-delayed condition (TD) shown in Figure 5 reveal a consistent decrease in RT as the target segments are delayed. Also apparent is the effect of noise type which nicely replicates the ordering obtained in the LI and ND conditions. An analysis of variance revealed significant effects of SOA $[F(4,12)=15.81, p<.001]$, noise $[F(4,16)=$ 14.79, $\mathrm{p}<.001]$, and SOA by Noise $[F(16,48)=$ $10.79, \mathrm{p}<.001]$.

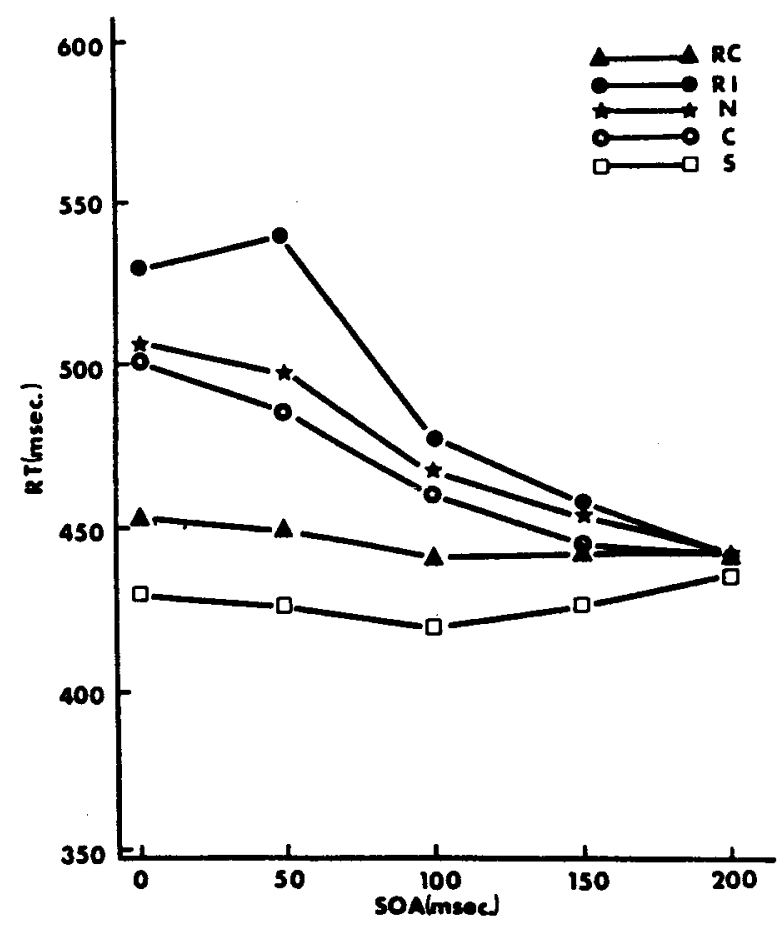

Figure 4. Mean reaction time as a function of SOA of the display and noise element segments. Parameter is noise type.

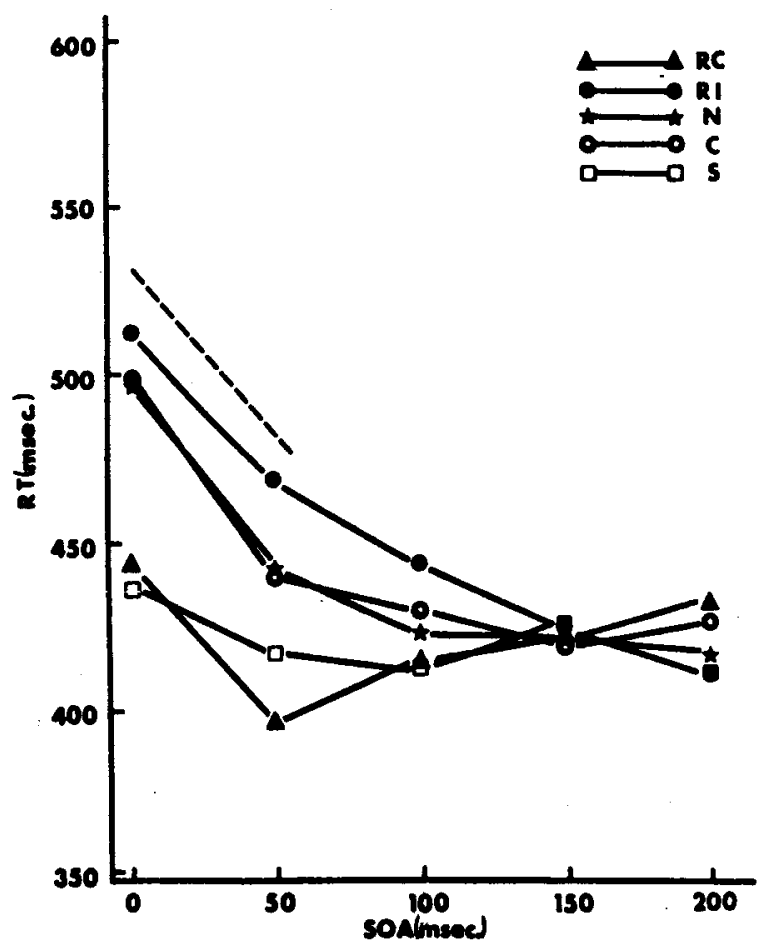

Figurs 3. Mean reaction time as a function of the SOA of display and turget eegments. Parameter nolise type. Dabed Ine correaponds to a slope $=-1$. 
Of particular interest is the form of the function relating RT to delay of the target segments for the different types of noise. As is apparent from Figure 5, all functions, with the exception of the single target, show a slope approximating -1 (designated by a dashed line in the figure) for the first $50 \mathrm{msec}$. Since the RTclock did not start until the complete target was present, this slope corresponds to the performance of a system that does not make use of the target features for the first $50 \mathrm{msec}$. That is, by starting the clock $50 \mathrm{msec}$ after display onset, we are subtracting $50 \mathrm{msec}$ dead time (insofar as the presence of a complete target is concerned). Thus it appears that the visual information processing system does not use the complete target or noise element information for the first $50 \mathrm{msec}$ when confronted with a multicharacter display. A single-character display evidently allows a quicker utilization of symbolic information.

Beyond $50 \mathrm{msec}$, functions depart from the -1 slope, indicating that target segments, if present, may now be utilized. At 150-msec SOA, noise elements no lunger slow the response to the target element. This is to be contrasted with the ND condition, in which a similar point was not reached until 200 -msec SOA, and the LI condition, where interference was found at all SOAs.

\section{DISCUSSION}

The present experiment provides support for the notion that a visual input is processed on several different levels. An observer confronted with a complex array of characters is indifferent to the presence of defining features of those characters for approximately $50 \mathrm{msec}$. Beyond this delay, the system is sensitive to complete forms in target and nontarget positions. This period of indifference, which may correspond to the operation of Neisser's (197) preattentive process, appears to depend on the complexity of the input, since a single-character display allowed utilization of a complete character at the 50-msec delay. Other investigators have recently reported that $\mathrm{RT}$ is indifferent for short time periods to the presence of complete information for individual letters in a word (Eriksen \& Eriksen, 1974a) and separate components which can be combined to yield horizontal or vertical stripes (Rohrbaugh \& Eriksen, 1975).

There are three major findings in the LI condition which require interpretation. First, providing the subject with information about the location of the target before the display appears decreases RT even when the target is not embedded in noise elements.

Second, the interference provided by nonindicated display elements depends on their response relationship to the target as well as target-noise physical similarity. Surrounding a target with its replicas produces no interference, while noise elements which belong to the opposite response set produce sizable increments in RT. This might suggest that response compatibility was the sole determiner of interference were it not for the intermediate deficits produced by elements which neither belong to a response set $(\mathrm{N})$ nor possess a verbal label (C).

Finally, the differences existing between various types of noise elements are attenuated but not eliminated by the leading indicator. This attenuation is primarily attributable to performance on the RI, N, and $C$ conditions approaching that of a single target (S) at long lead times while maintaining their own relative disparities, It is unlikely that greater lead times of the indicator would eliminate these differences, since previous research has shown that effects of response compatibility and siplay size are present even with asymptotic lead times (Colegate, Hoffman, \& Eriksen, 1973; Eriksen \& Hoffman, 1973).

These findings suggest that attention is not a simple attenuation of unattended inputs as Treisman (1960) has suggested for the auditory modality. The finding that recognition latency of a single target is decreased by providing location information suggests, rather, that attention may be an enhancement of the attended item. This could take the form of a higher rate of processing for attended items, as Eriksen and Hoffman (1973) have suggested, or possibly an actual increase in neural intensity, although this latter possibility is unlikely in view of Sokolov's (1960) finding that dishabituation may occur in response to a signal decrement as well as increment.

Whatever form attention takes, it is not perfectly effective in eliminating the interfering effects of noise elements. This may be due to a scanning mechanism which has an irreducible size so that elements adjacent to the target also receive processing. This effect is now well established for a variety of tasks (Eriksen \& Eriksen, 1974b; Eriksen \& Hoffman, 1972, 1973; Holmgren, 1974). The nature of this processing, however, is not clear.

Estes (1972) has proposed that adjacent elements may interfere with each other by sharing the same processing channeis or by mutual inhibition of feature detectors. Application of such a model to the present results would be difficult but not impossible. One need only postulate that the activity of feature detectors associated with the two possible targets (3 and 5) have lower thresholds than other detectors (Estes, 1972). Thus, the feature detectors in positions adjacent to the target would be more active when processing one of the target elements. By invoking the additional ssumption that the inhibition which a detector exerts on a neighboring detector is proportional to its own rate of activity, we can account for the difference between the RI and the $C$ and $N$ conditions. This assumption is a plausible one since 
recurrent lateral inhibitory nets, such as are found in the eye of Limulus, display precisely this property (Cornsweet, 1970). The equivalence of the RC and S conditions, however, would have to be accounted for in an ad hoc fashion, perhaps by postulating that channels going to the same detector do not have inhibitory connections.

Two considerations argue against this extension of the Estes (1972) model. One is the high error rate $(8 \%)$ obtained in the RI condition compared to other conditions $(1 \%-2 \%)$. It is unlikely that feature inhibition alone would produce such a high error rate with the energy levels employed in this study. Rather, it appears that interference is occurring on a much higher level, one responsible for loading and executing the motor programs required for response. This suggests that noise elements undergo a considerable degree of analysis.

The second piece of evidence arguing against a feature inhibition explanation is the finding that as the target segments are delayed, noise elements exert a declining effect until, at $150-\mathrm{msec}$ SOA, they no longer produce interference. The feature inhibition approach could encompass this finding by proposing that inhibitory effects decay by $150 \mathrm{msec}$. This modification would not likely be invoked, however, in view of Townsend, Taylor, and Brown's (1971) finding that adjacent letters may mask one another even under conditions of unlimited viewing time.

An alternative approach, the one favored in this paper, stresses the visual system's ability to detect redundancy in its in put. Inspection of Figure 2 reveals that the RC condition is the only one in which the display was completely homogeneous. Assume that homogeneity or identity can be detected early in the processing sequence, perhaps by the preattentive stage posited by Neisser (1967). In the RI, N, and C conditions, the information that a different display element was present would be available before the identity of that element was known.

Determination of identity would be the task of a focal attentive stage, perhaps taking the form of an internal scan as proposed by Eriksen and Hoffman (1973). Location of the indicated element would proceed via a process of feedback so that elements in close proximity to the target would also be scanned. When the display is homogeneous, however, the scanner need only sample one display element to determine the identity of the target.

The decision of what elements are to be scanned and in what order can be viewed as a program directing the operation of the scanner. Construction of such a program would require time. The leading indicator, then, allows this program to be constructed before the display appears, with a resulting savings in RT.

The role of the scanning mechanism is presumably one of pattern recognition. This may be accomplished by synthesizing the features present to form a schematic description of the object (Neisser, 1967). Supporting evidence for this view is provided by the data of Beck and Ambler (1973). Subjects were required to discriminate an $L$ or tilted $T$ from an upright $T$ embedded in a display of upright Ts. When attention was distributed, discriminability of the tilted $T$ was superior to that of the $L$. As the authors suggest, the preattentive mechanism can group objects on the basis of line tilt but not line arrangement (at least for peripheral vision). When the target location was provided $150 \mathrm{~m}: \mathrm{c}$ before the display appeared, discrimination of the targets was approximately equal. Presumably, the indicator caused the subjects to switch from a level that included the entire display. to one that included a single object.

The present results suggest that the operation of synthesizing an object may be a serial operation which slows subsequent synthesis of the target. Object synthesis may consist of placing a schematic representation of the object into a more permanent form of visual memory. Further processing of the object may be conducted, resulting in the assignment of verbal labels and preparation of response programs. In the case of response-incompatible noise elements, the latter operation must be inhibited which would require time as well as providing the opportunity for occasional execution leading to an error. Thus, noise elements may interfere on two levels: a schematic encoding followed by response preparation.

Support for a feedback mechanism underlying attention is provided by the TD condition. When the target segments are presented at $150-\mathrm{msec}$ SOA, the noise elements no longer interfere. This suggests that the scanning mechanism has had time to precisely locate the target element, since a partially completed form was present in the target location. This advantage does not exist with the leading indicator, so some interference is always present. The ND condition, on the other hand, does not show cessation of interference until a 200-msec delay of the segments. We can speculate that, by 150 -msec SOA, the subject is indeed locked onto the target. However, the sudden occurrence of new features in unattended display locations may cause a brief interrupt of the scanning mechanism, with a resultant slowing of target processing.

\section{CONCLUSIONS}

The present experiment provides evidence for a hierarchically organized processing system. An initial preattentive stage seeks an overall or global organization of the input, a particularly important form of which is redundancy. This stage is also responsible for constructing a program to guide the 
operation of a second stage, which has been likened to an internal scanner. This scanner is responsible for the abstraction of a schematic description of the object in its tield. The encoding process is a serial one that may interfere with the later encoding of subsequently scanhed objects.

Attention is viewed as a feedback-controlled process so that elements adjacent to the target may also be encoded. If the display is homogeneous, only a single element need be scanned. For a heterogeneous display, however, precise localization is necessary so that (1) a feedback-controlled location of the target is initiated and (2) noise elements may be encoded. If noise elements are encoded, they provide interference due to the usage of a serial encoding stage as well as the initiation of response stages if they are members of a response set.

This characterization of visual processing does not deny the role of signal-noise confusability that forms the cornerstone of the unlimited capacity models of Gardner (1973), Kinchla (1974), and Shiffrin and Geisler (1973). It merely suggests that the many spatial and temporal interactions reviewed in this paper implicate an important role both for the Gestalt structure present in visual displays and the ability of attention to control which display elements are encoded.

\section{REFERENCES}

BECK, J., \& AMBLER, B. The effects of concentrated and distributed attention on peripheral acuity. Perception \& Psychophysics, 1973, 14, 225-230.

Colegate, R. L., Hoffman, J. E.. \& Eriksen, C. W. Selective encoding from multielement visual displays. Perception \& Psychophysics, 1973, 14, 217-224.

Cornsweet, T. N. Visual perception. New York: Academic Press, 1970.

Eruxsen, B. A., \& Eruxsen, C. W. The importance of being first: A tachistoscopic study of the contribution of each letter to the recognition of four-letter words. Penception \& Psychophysics, 1974, 15, 66-72. (a)

ERIKsin, B. A., \& Eniksen, C. W. Effects of noise letters upon the identification of a target letter in a nonsearch task. Penception \& Psychophysics, 1974, 16, 143-149. (b)

Eriksen, C. W., \& Horfman, J. E. Temporal and spatial characteristics of selective encoding from visual displays. Perception \& Psychophysics, 1972, 12, 201-204.

Eersen, C. W., \& Horman, J. E. The extent of processing of noise elements during selective encoding from visual displays. Perception \& Psychophysics, 1973, 14, 217-224.
Eriksen, C. W., \& Rohrbaugh, J. W. Some factors determining efficiency of selective attention. American Joumal of Psychology. 1970. 83. 330-342.

ERIKsen, C. W., \& SPencer, T. Rate of information processing in visual perception: Some results and methodological considerations. Journal of Experimental Psychology Monograph, 1969, 79, 1-16.

Estes, W. K. Interactions of signal and background variables in visual processing. Perception \& Psychophysics, 1972, 12, 278-286.

GARDNER, G. T. Evidence for independent parallel channels in tachistoscopic perception. Cognitive Psychology. 1973, 4. 130-155.

Guzman, A. Decomposition of a visual scene into three-dimensional bodies. In A. Grasselli (Ed.), Automatic interpretation and classification of images. New York: Academic Press. 199.

Holmgren. J. E. The effect of a visual indicator on rate of visual search: Evidence for processing control. Perception \& Psychophysics, 1974, 15, 544-550.

Kinchla, R. A. Detecting target elements in multi-element arrays: A confusability model. Perception \& Psychophysics, 1974, 15, 149-158.

NeIsser, T. Cognitive psychology. New York: AppletonCentury-Crofts. $1 \% 67$.

Rohrbaugh, J. W., \& Eruksen, C. W. Reaction time measurement of temporal integration and organization of form. Perception \& Psychophysics, 1975, 17, 53-58.

Shiffrin, R. M., \& GARDner, G. T. Visual processing capacity and attentional control. Joumal of Experimental Psychology, 1972, 93. 72-82.

Shiffrin, R. M., \& Geisler, W. S. Visual recognition in a theory of information processing. In R. Solso (Ed.), The Loyola Symposium: Contemponary viewpoints in cognitive psychology. Washington: Winston, 1973.

Soxolov, E. N. Neuronal models and the orienting reflex. In M. A. B. Brazier (Ed.), The central nerwous system and behavior. New York: Josiah Macy, Jr., Foundation, $1 \% 60$.

SPENCER, T. J. Some effects of different masking stimuli on iconic storage. Joumal of Experimental Psychology, 1969, 81, 132-140.

SperLing, G. The information available in brief visual presentations. Psychological Monographs, 1960, 74, No. 11.

Townsend, J. T.. TAylor, S. G., \& Brown, D. R. Lateral masking for letters with unlimited viewing time. Perception \& Psychophysics. 1971, 10. 375-378.

Treisman, A. M. Contextual cues in selective listening. Quarterly Joumal of Experimental Psychology, 1960, 12 , 242-248.

(Received for publication April 16, 1975; revision received July 7,1975 .) 